\title{
Evolution equations with dynamic boundary conditions
}

\author{
Hintermann, Thomas
}

\begin{abstract}
SynopsisIn this paper, we study boundary problems with dynamic boundary conditions, that is, with boundary operators containing time derivatives. The equations under consideration are transformed into abstract Cauchy problems $<$ jats:italic $>\mathrm{x}</$ jats:italic $>-<$ jats:italic $>\mathrm{Cx}</$ jats:italic $>=<$ jats:italic $>$ f $</$ jats:italic $>$ an $=<$ jats:italic $>\mathrm{x}</$ jats:italic $><$ jats:sub $>0</$ jats:sub $>$. Abstract theoretical results concerning the operators $<$ jats:italic $>\mathrm{C}</$ jats:italic $>$ are obtained by the study of a naturally arising pseudodifferential operator. For existence and uniqueness theorems concerning solutions of parabolic and hyperbolic equations, we then apply the theory of semigroups in Banach spaces. Some examples of semilinear and quasilinear problems, to which our results apply, are given.
\end{abstract}

DOI: https://doi.org/10.1017/s0308210500023945

Posted at the Zurich Open Repository and Archive, University of Zurich ZORA URL: https://doi.org/10.5167/uzh-154924

Journal Article

Published Version

Originally published at:

Hintermann, Thomas (1989). Evolution equations with dynamic boundary conditions. Proceedings of the Royal Society of Edinburgh Section A: Mathematics, 113(1-2):43-60.

DOI: https://doi.org/10.1017/s0308210500023945 


\title{
Evolution equations with dynamic boundary conditions
}

\author{
Thomas Hintermann \\ Mathematisches Institut der Universität Zürich, Rämistrasse 74, CH-8001 Zürich, \\ Switzerland
}

(MS received 17 August 1988. Revised MS received 22 March 1989)

\section{Synopsis}

In this paper, we study boundary problems with dynamic boundary conditions, that is, with boundary operators containing time derivatives. The equations under consideration are transformed into abstract Cauchy problems $\dot{x}-C x=f$ and $x(0)=x_{0}$. Abstract theoretical results concerning the operators $C$ are obtained by the study of a naturally arising pseudodifferential operator. For existence and uniqueness theorems concerning solutions of parabolic and hyperbolic equations, we then apply the theory of semigroups in Banach spaces. Some examples of semilinear and quasilinear problems, to which our results apply, are given.

\section{Introduction}

In this paper, we study differential equations with boundary conditions containing time derivatives. Let $\Omega$ be a bounded domain in $\mathbb{R}^{n}$ with smooth boundary $\partial \Omega$ and outer unit normal vector field $v$, and let $\Delta$ be the Laplace operator with respect to the space variables. By dots we denote time derivatives and the given functions $f$ and $g$ may depend on space, time or the solutions. We look for existence and uniqueness results about equations of the following types:

(P1)

$$
\begin{aligned}
& \dot{u}-\Delta u=f \quad \text { in } \Omega \times \mathbb{R}_{+}, \\
& \dot{u}+\frac{\partial u}{\partial v}=g \quad \text { on } \partial \Omega \times \mathbb{R}_{+} \text {, } \\
& u(0)=u_{0} \quad \text { in } \bar{\Omega} \text {. } \\
& \text { (P2) }-\Delta u=f \quad \text { in } \Omega \times \mathbb{R}_{+} \text {, } \\
& \dot{u}+\frac{\partial u}{\partial v}=g \quad \text { on } \partial \Omega \times \mathbb{R}_{+} \text {, } \\
& u(0)=u_{0} \quad \text { on } \partial \Omega \text {. } \\
& \text { (P3) }-\Delta u=f \quad \text { in } \Omega \times \mathbb{R}_{+} \text {, } \\
& \ddot{u}+\frac{\partial u}{\partial v}=g \quad \text { on } \partial \Omega \times \mathbb{R}_{+}, \\
& u(0)=u_{0} \text { on } \partial \Omega \text {, } \\
& \dot{u}(0)=u_{1} \quad \text { on } \partial \Omega \text {. }
\end{aligned}
$$

Problems of type (P1) are already mentioned in [9, appendix to par. 3]. We find them again in heat transfer problems in a solid in contact with a fluid [18]. In some recent publications, Sauer [22] and Grobbelaar-van Dalsen [14] treat heat 
transfer problems with dynamic boundary conditions by means of their theory of "B-evolutions", which is applied to some semilinear and quasilinear problems in Hilbert spaces.

Problem (P3) sometimes occurs in connection wtih gravity waves ([17, Chapter 9]). Some cases are treated by Garipov [12] and Friedman and Shinbrot [11]. The latter authors also briefly discuss (P2).

Lions [19] and Diaz and Jimenez [10] use the theory of maximal monotone operators to solve some special nonlinear equations of types (P2) and (P3). Related problems with dynamic boundary conditions have been considered by Gröger (e.g. [13]) and Van Rensburg [26]; Rossouw [21] studied the physicomathematical origin of these conditions. Lewis, Marsden and Ratiu [27] and Okamoto [28] use the Hamiltonian structure of perfect fluid dynamics to investigate free boundary problems arising in this context (cf. also [29]).

In our approach to the solution of such problems, we use the theory of semigroups in Banach spaces. To give an idea of our methods, let us look at the formally easiest problem (P2). Observe that the operator of interest, here the Neumann operator, maps functions defined on the domain $\Omega$ to functions defined on its boundary $\partial \Omega$, and therefore it is not a candidate for a generator of a semigroup on any function space. To make semigroup theory applicable, our problem must first be transformed.

We now use the notation $A=-\Delta$ and $B_{0}=(u \mapsto u \mid \partial \Omega)$ and assume that the Dirichlet problem $-\Delta u(x)=f(x)$ in $\Omega, u(x)=g(x)$ on $\partial \Omega$ is uniquely solvable, that is, $\left(A, B_{0}\right) \in$ isom $(X, Y \times Z)$ for suitable function spaces $X, Y, Z$. Thus we can decompose the space of solutions $X$ into the direct sum

$$
\begin{aligned}
X & =\operatorname{ker} B_{0} \oplus \operatorname{ker} A, \\
u & =\left(A, B_{0}\right)^{-1}(A u, 0)+\left(A, B_{0}\right)^{-1}\left(0, B_{0} u\right) \\
& =: w+R_{0} B_{0} u \\
& =: w+R_{0} z .
\end{aligned}
$$

Setting $B:=\partial / \partial v,(\mathrm{P} 2)$ is equivalent to $A w=f$ in $\Omega \times \mathbb{R}_{+}, \dot{z}+B R_{0} z=g-B w=$ $g-B\left(A, B_{0}\right)^{-1}(f, 0)$ on $\partial \Omega \times \mathbb{R}_{+}$and $z(0)=z_{0}$ on $\partial \Omega$. By obvious identifications, we now rewrite the last two lines as an abstract nonlinear Cauchy problem $\dot{x}-C x=F(x), x(0)=x_{0}$, and so the natural question arises as to whether the operator $C:=-B R_{0}$ is the generator of a semigroup. This question has already been formulated in [19]. In the first part of this paper, we give a rather general and positive answer, in that we allow much more general operators than the Laplacian and the Neumann boundary operator.

The difficult part of the proof is the derivation of resolvent estimates. With this aim, we represent $B R_{0}$ for the case $\Omega$ being the halfspace $\mathbb{R}^{n-1} \times \mathbb{R}_{+}$as a pseudodifferential operator, that is, $B R_{0}=\mathscr{F}_{n-1}^{-1} \mathbb{B R}_{0}\left(\xi^{\prime}\right) \mathscr{F}_{n-1}$, where we denote the Fourier transform in $\mathbb{R}^{n-1}$ by $\mathscr{F}_{n-1}$. The symbol $\mathbb{B R}_{0}\left(\xi^{\prime}\right)$, a complex $N \times N$-matrix-valued map of $\mathbb{R}^{n}$, possesses certain homogeneity properties with respect to the variables $\xi^{\prime}$, which enables us to apply the Michlin-Hörmander multiplier theorem. Once we have established the desired estimate in the halfspace, we carry it over to the general situation by the well-known Korn trick which involves localisation and perturbation arguments. 
We then transform the problems under consideration as indicated above, and apply some results from nonlinear functional analysis. There we profit from the precise knowledge of the domains of the operators involved. Since we can work in $L_{p}$-spaces, we can allow quite general nonlinearities. This paper contains a summary of the author's doctoral thesis, which we refer to for additional information about examples and details of proofs omitted or shortened here.

\section{A priori estimates}

\subsection{Definitions and assumptions}

Let $n, m, N \in \mathbb{N} \backslash\{0\}, n \geqq 2$, and let $\Omega \subset \mathbb{R}^{n}$ be a bounded domain with smooth boundary $\partial \Omega$. Given $a_{\alpha}: \Omega \rightarrow \mathscr{L}\left(\mathbb{C}^{N}\right), \alpha \in \mathbb{N}^{n},|\alpha| \leqq 2 m$, and $b_{\beta}^{j}: \partial \Omega \rightarrow \mathscr{L}\left(\mathbb{C}^{N}, \mathbb{C}\right)$, $\beta \in \mathbb{N}^{n}, \quad|\beta| \leqq c_{j}, \quad 0 \leqq c_{j} \leqq 2 m-1, \quad j=1, \ldots, m N$, we form the differential operators

$$
\begin{gathered}
\mathscr{A}(x, D):=\sum_{|\alpha| \leqq 2 m} a_{\alpha} D^{\alpha}, \\
\mathscr{B}^{j}(x, D):=\sum_{|\beta| \leqq c_{j}} b_{\beta}^{j} D^{\beta}, \\
\mathscr{B}(x, D):=\left(\mathscr{B}^{1}(x, D), \ldots, \mathscr{B}^{m N}(x, D)\right),
\end{gathered}
$$

where the boundary operators are always understood in the trace sense. We denote the principal parts by $\mathscr{A}_{H}(x, D)$ and $\mathscr{B}_{H}^{j}(x, D)$, respectively.

Throughout this paper, the system $(\mathscr{A}(x, D), \mathscr{B}(x, D))$ of operators is assumed to have the following properties (cf. [7]):

(A) $\mathscr{A}(x, D)$ is normally elliptic, that is $\sigma\left(\mathscr{A}_{H}(x, \xi)\right) \subset[$ re $z>0]:=$ $\{z \in \mathbb{C} \mid$ re $z>0\}$ for all $(x, \xi) \in \bar{\Omega} \times \mathbb{S}^{n-1}$. Here we denote by $\sigma\left(\mathscr{A}_{H}(x, \xi)\right)$ the set of eigenvalues of the complex $N \times N$-matrix $\mathscr{A}_{H}(x, \xi)$, where $\mathscr{A}_{H}(x, \xi)$ is the symbol of $\mathscr{A}_{H}(x, D)$.

Let $\mathscr{B}_{0}(D):=\left(1_{N}, i(\partial / \partial v) 1_{N}, \ldots,(i(\partial / \partial v))^{m-1} 1_{N}\right)$ be the Dirichlet operator, where $v$ is the outer unit normal vector field on $\partial \Omega$ and let $T(\partial \Omega)$ be the tangent bundle of $\partial \Omega$.

(B) $\mathscr{B}(x, D)$ satisfies the uniform strong complementing condition with respect to $\mathscr{A}(x, D)$, that is: For all $(x, \xi) \in T(\partial \Omega)$ and $\lambda, \mu \in[$ re $z \geqq 0]$ with $(\xi, \lambda) \neq(0,0)$ and $(\xi, \mu) \neq(0,0)$, the zero function is the only exponentially decaying solution of the system of ordinary differential equations

$$
\begin{aligned}
{\left[\lambda+\mathscr{A}_{H}\left(x, \xi-v(x) D_{t}\right)\right] u(t) } & =0, \quad t>0, \\
{\left[\mu \mathscr{B}_{0}+\mathscr{B}_{H}\left(x, \xi-v(x) D_{t}\right)\right] u(0) } & =0 .
\end{aligned}
$$

Additionally, the operator $\mathscr{B}_{0}$ is supposed to satisfy the normal complementing condition with respect to $\mathscr{A}$, that is, we have (B) for $\mathscr{B}_{0}$ with $\mu=0$.

Remarks 1.1. (i) (A) is implied by the uniform strong ellipticity, that is, re $\left(\mathscr{A}_{H}(x, \xi) \eta \mid \eta\right) \geqq \alpha>0$ for all $(x, \xi) \in \Omega \times \mathbb{S}^{n-1}, \eta \in \mathbb{C}^{N},|\eta|=1$.

(ii) (A) implies the strong root condition: For all $(x, \xi) \in T(\partial \Omega), \lambda \in[$ re $z \geqq 0]$, $(\lambda, \xi) \neq(0,0)$ the polynomial $\left(z \mapsto \operatorname{det}\left(\lambda+\mathscr{A}_{H}(x, \xi-v(x) z)\right)\right)$ has exactly $m N$ roots in [im $z>0]$. 
EXAMPLES 1.2. The assumptions are fulfilled in the case of a uniformly very strongly elliptic second order system (i.e. the differential operator satisfies the uniform Legendre condition) with mixed conormal and Dirichlet boundary conditions and for certain systems of separated divergence form (cf. [7]).

\subsection{Representation of the pseudodifferential operator}

In this section, we assume that the operators have constant coefficients and derivatives of highest order only, that is,

$$
\begin{aligned}
& \mathscr{A}(D):=\sum_{|\alpha|=2 m} a_{\alpha} D^{\alpha}, \quad a_{\alpha} \in \mathscr{L}\left(\mathbb{C}^{N}\right), \quad|\alpha|=2 m, \\
& \mathscr{B}^{j}(D):=\sum_{|\beta|=c_{j}} b_{\beta}^{j} D^{\beta}, \quad b_{\beta}^{j} \in \mathscr{L}\left(\mathbb{C}^{N}, \mathbb{C}\right), \quad|\beta|=c_{j}, \quad j=1, \ldots, m N .
\end{aligned}
$$

Let $S_{\alpha}:=\{z \in \mathbb{C}|| \arg z \mid \leqq \alpha\} \cup\{0\}$ and $\mathbb{R}_{+}:=(0, \infty)$. We choose $\eta \in S_{\pi / 4 m}$ satisfying $\eta^{2 m}=\lambda$ and consider the problem

$$
\begin{aligned}
(\lambda+\mathscr{A}(D)) u\left(x^{\prime}, t\right) & =0 & & \text { in } \mathbb{R}^{n}:=\mathbb{R}^{n-1} \times \mathbb{R}_{+}, \\
\mathscr{B}^{j}(D) u\left(x^{\prime}, 0\right) & =g^{j}\left(x^{\prime}\right) & & \text { on } \mathbb{R}^{n-1}, j=1, \ldots, m N,
\end{aligned}
$$

where $g^{j} \in \mathscr{S}\left(\mathbb{R}^{n-1}, \mathbb{C}\right)$, the Schwartz space of rapidly decreasing functions. Applying the Fourier transform with respect to the variable $x^{\prime}$, and fixing $\xi^{\prime} \in \mathbb{R}^{n-1} \equiv \mathbb{R}^{n-1} \times\{0\} \subset \mathbb{R}^{n}$, we are led to the system of ordinary differential equations of order $2 m$

$$
\begin{aligned}
\left(\lambda+\mathscr{A}\left(\xi^{\prime}+e_{n} D_{t}\right)\right) \hat{u}\left(\eta, \xi^{\prime}, t\right) & =0 & & \text { in } \mathbb{H}^{n}, \\
\mathscr{B}^{\prime}\left(\xi^{\prime}+e_{n} D_{t}\right) \hat{u}\left(\eta, \xi^{\prime}, 0\right) & =\hat{g}^{j}\left(\xi^{\prime}\right) & & \text { in } \mathbb{R}^{n-1}, j=1, \ldots, m N,
\end{aligned}
$$

where $e_{n}:=(0, \ldots, 1)$.

For each $z \in \mathbb{C}$ we define $\mathscr{A}\left(\xi^{\prime}+e_{n} z\right)=: \sum_{k=0}^{2 m} a_{2 m-k}\left(\xi^{\prime}\right) z^{k}$, where, obviously,

$$
a_{2 m-k}\left(r \xi^{\prime}\right)=r^{2 m-k} a_{2 m-k}\left(\xi^{\prime}\right) \quad \forall\left(\xi^{\prime}, r\right) \in \mathbb{R}^{n-1} \times \mathbb{R}_{+},
$$

and because of (A) we have $a_{0}=\mathscr{A}\left(e_{n}\right) \in \mathscr{G} \mathscr{L}\left(\mathbb{C}^{N}\right)$, the group of invertible complex $N \times N$-matrices. Moreover, we put $\mathscr{R}^{j}\left(\xi^{\prime}+e_{n} z\right)=: \sum_{l=0}^{c_{i}} b_{j, c_{j}-l}\left(\xi^{\prime}\right) z^{l}$, where

$$
b_{j, c_{j}-l}\left(r \xi^{\prime}\right)=r^{c_{j}-l} b_{j, c_{j}-l}\left(\xi^{\prime}\right) \quad \forall\left(\xi^{\prime}, r\right) \in \mathbb{R}^{n-1} \times \mathbb{R}_{+}, \quad j=1, \ldots, m N .
$$

The expressions

$$
\begin{aligned}
\tilde{a}_{j} & :=\tilde{a}_{j}\left(\xi^{\prime}\right):=-a_{0}^{-1} a_{j}\left(\xi^{\prime}\right), \quad j=1, \ldots, 2 m-1, \\
\tilde{a}_{2 m} & :=\tilde{a}_{2 m}\left(\eta, \xi^{\prime}\right):=-a_{0}^{-1}\left(\eta^{2 m}+a_{2 m}\left(\xi^{\prime}\right)\right),
\end{aligned}
$$

are then used to define the matrix-valued function

$$
\mathbb{A}:=\left[\begin{array}{cccccc}
0 & 1 & & & & \\
& 0 & 1 & & & \\
& & \ddots & \ddots & & \\
& & & \ddots & \ddots & \\
& & & & 0 & 1 \\
\tilde{a}_{2 m} & \tilde{a}_{2 m-1} & \cdots & \cdots & \cdots & a_{1}
\end{array}\right]: S_{\pi / 4 m} \times \mathbb{R}^{n-1} \rightarrow \mathscr{L}\left(\mathbb{C}^{2 m N}\right) .
$$


For every $\xi^{\prime} \in \mathbb{R}^{n-1}$, let $\mathbb{B}\left(\xi^{\prime}\right) \in \mathscr{L}\left(\mathbb{C}^{2 m N}, \mathbb{C}^{m N}\right)$ be the matrix with rows

$$
\begin{aligned}
\mathbb{B}\left(\xi^{\prime}\right):= & {\left[b_{j, c_{j}}\left(\xi^{\prime}\right), b_{j, c_{j}-1}\left(\xi^{\prime}\right), \ldots, b_{j, 0}\left(\xi^{\prime}\right), 0, \ldots, 0\right] } \\
& \in \mathscr{L}\left(\mathbb{C}^{2 m N}, \mathbb{C}\right), \quad c_{i} \leqq c_{j} \quad \forall i \leqq j, \quad i, j=1, \ldots, m N .
\end{aligned}
$$

Finally, we put

$$
\begin{aligned}
v & :=\left(v_{1}, \ldots, v_{2 m}\right)^{\top} \in \mathbb{C}^{2 m N}, \\
v_{k} & :=v_{k}\left(\eta, \xi^{\prime}, t\right):=D_{t}^{k-1} \hat{u}\left(\eta, \xi^{\prime}, t\right), \quad k=1, \ldots, 2 m, \quad\left(\xi^{\prime}, t\right) \in \mathbb{H}^{n}, \\
h & :=\hat{g}:=\left(\hat{g}_{1}, \ldots, \hat{g}_{m N}\right)^{\top} \in \mathscr{S}\left(\mathbb{R}^{n-1}, \mathbb{C}^{m N}\right) .
\end{aligned}
$$

Then (1.3), (1.4) is equivalent to the first order system

$$
\begin{aligned}
D_{t} v\left(\eta, \xi^{\prime}, t\right) & =\mathbb{A}\left(\eta, \xi^{\prime}\right) v\left(\eta, \xi^{\prime}, t\right), \\
\mathbb{B}\left(\xi^{\prime}\right) v\left(\eta, \xi^{\prime}, 0\right) & =h\left(\xi^{\prime}\right), \quad\left(\xi^{\prime}, t\right) \in \mathbb{T}^{n} .
\end{aligned}
$$

LEMMA 1.3 .

$$
\operatorname{det}\left(\eta^{2 m}+\sum_{k=0}^{2 m} a_{2 m-k} \lambda^{k}\right)=\operatorname{det}\left(a_{0}\right) \operatorname{det}(\lambda-\mathbb{A}) \text {. }
$$

Proof. The claim follows by expansion with respect to the last $N$ rows of the determinant of $\lambda-A$ and elementary matrix manipulations [15].

As implied by the statement above, the roots of the polynomial $(z \mapsto \operatorname{det}(\lambda+$ $\left.\mathscr{A}\left(\xi^{\prime}+e_{n} z\right)\right)$ ) are exactly the eigenvalues of $\mathbb{A}\left(\eta, \xi^{\prime}\right)$. Hence, by the root condition (Remark 1.1(ii)), $e^{i \mathbb{A}\left(\eta, \xi^{\prime}\right) t}$ is a linear hyperbolic flow [3], the stable (unstable) subspace of which we denote by $E_{+}\left(\eta, \xi^{\prime}\right)\left(E_{-}\left(\eta, \xi^{\prime}\right)\right)$.

Remarks 1.4. (i) $E_{+}\left(\eta, \xi^{\prime}\right)$ (respectively $E_{-}\left(\eta, \xi^{\prime}\right)$ ) is the direct sum of the algebraic eigenspaces belonging to the eigenvalues of $i A$ with real part in the left (right) complex half plane [3, Theorem (13.4)].

(ii) As an obvious consequence of the root condition (Remark 1.1(ii)) we have

$$
\operatorname{dim} E_{+}\left(\eta, \xi^{\prime}\right)=\operatorname{dim} E_{-}\left(\eta, \xi^{\prime}\right)=m N, \quad \xi^{\prime} \in \mathbb{R}^{n-1} .
$$

(iii) The operator $\mathscr{B}$ satisfies the normal complementing condition if and only if $\mathbb{B}\left(\xi^{\prime}\right) \in \operatorname{isom}\left(E_{+}\left(\eta, \xi^{\prime}\right), \mathbb{C}^{m N}\right)$. This follows easily from the fact that the solutions of $(1.3)$ are given by the formula $\hat{u}\left(\eta, \xi^{\prime}, t\right)=p r_{1}\left(e^{i \mathbb{A}\left(\eta, \xi^{\prime}\right) t} v\left(\eta, \xi^{\prime}, 0\right)\right)$, where $p r_{1}(v):=v_{1}$ for $v=\left(v_{1}, \ldots, v_{2 m}\right) \in \mathbb{C}^{2 m N}$.

For the operator $\mathscr{B}_{0}(D)=\left(1_{N}, D_{t} 1_{N}, \ldots, D_{t}^{m-1} 1_{N}\right)$ we have $\mathbb{B}_{0}\left(\xi^{\prime}\right)=$ $\left[1_{m N}, 0\right] \in \mathscr{L}\left(\mathbb{C}^{2 m N}, \mathbb{C}^{m N}\right)$, for all $\xi^{\prime} \in \mathbb{R}^{n-1}$. Because of our assumption (B) and Remark 1.4(iii) we can define the operator $\mathbb{B}_{0}\left(\eta, \xi^{\prime}\right):=\left(\mathbb{B}_{0} \mid E_{+}\left(\eta, \xi^{\prime}\right)\right)^{-1} \in$ isom $\left(\mathbb{C}^{m N}, E_{+}\left(\eta, \xi^{\prime}\right)\right)$ and also the matrix-valued function $\mathbb{B R}_{0}\left(\eta, \xi^{\prime}\right):=$ $\mathbb{B}\left(\xi^{\prime}\right) \mathbb{R}_{0}\left(\eta, \xi^{\prime}\right)$.

The heart of this section is the following lemma.

LemMA 1.5. (i) $\mu+\mathbb{B R P}\left(\eta, \xi^{\prime}\right) \in \mathscr{G} \mathscr{L}\left(\mathbb{C}^{m N}\right)$ for all $\mu \in[\operatorname{Re} \mu \geqq 0]$.

(ii) $\left[\mathbb{B R}_{0}\left(\eta, \xi^{\prime}\right)\right]_{k, l+1} \in \mathscr{L}\left(\mathbb{C}^{N}, \mathbb{C}\right)$ is positively homogeneous of degree $c_{k}-l$ $k=1, \ldots, m N, l=0, \ldots, m-1$.

Proof. Assumption (B) and Remark 1.4(iii) imply (i). In the case of a single equation $(N=1)$, the proof of (ii) becomes much easier and, apart from simple 
alterations, can be found in [25]. In the system case, we decompose the matrix $A\left(\eta, \xi^{\prime}\right)$ in canonical Jordan form, which leads to a useful matrix representation of $\mathbb{R}_{0}\left(\eta, \xi^{\prime}\right)$. The homogeneity property (ii) then follows from (1.5) and (1.6). For details see Hintermann [15].

In the sequel, we always refer to the notations

$$
b:=\left(b_{1}, \ldots, b_{m N}\right):=(\overbrace{0, \ldots, 0,1, \ldots, 1, \ldots, m-1, \ldots, m-1)}^{N \text { times }}
$$

and

$$
c:=\left(c_{1}, \ldots, c_{m N}\right) \in \mathbb{N}^{m N} .
$$

The vector $b$ (respectively $c$ ) gives the order of the operator $\mathscr{B}_{0}$ (respectively $\mathscr{B}$ ).

Remarks 1.6. (i) As the proof of Lemma 1.5(ii) shows, we always have $\left[\mathbb{B} \mathbb{R}_{0}\left(\eta, \xi^{\prime}\right)\right]_{i j}=0$ if $c_{i}-b_{j}>0,1 \leqq i, j \leqq m N$.

(ii) We may always assume $c \geqq b$. Indeed, if $c_{i}<b_{i}, i \in\{1, \ldots, m N\}$, then $c_{j} \leqq b_{i}$ for all $j \leqq i$ and so, by the construction of $\mathbb{B}\left(\xi^{\prime}\right)$, its rank is less than maximal and $\mathbb{B R}_{0}\left(\eta, \xi^{\prime}\right)$ is not invertible.

As a summary of this section, we have the following conclusions: for any $g$ in, say, the space of tempered distributions, the Dirichlet problem $(\lambda+\mathscr{A}(D)) u=0$ in $\mathbb{H}^{n}, \mathscr{B}_{0}(D) u(0)=g$ on $\mathbb{R}^{n-1}$ has a solution given by the formula $u:=\mathscr{R}_{0}(\lambda) g=$ $p r_{1} \mathscr{F}_{n-1}^{-1} e^{i A\left(\eta, \xi^{\prime}\right) t} \mathbb{R}_{0}\left(\eta, \xi^{\prime}\right) \mathscr{F}_{n-1} g$. The operator $\mathscr{B} \mathscr{R}_{0}(\lambda): g \mapsto \mathscr{B}(D) \mathscr{R}_{0}(\lambda) g$ is a pseudodifferential operator, and its symbol is the matrix-valued function $\mathbb{B R}_{0}\left(\eta, \xi^{\prime}\right)$, that is, $\mathscr{B} \mathscr{R}_{0}(\lambda) g=\mathscr{F}_{n-1}^{-1} \mathbb{B R}_{0}\left(\eta, \xi^{\prime}\right) \mathscr{F}_{n-1} g$.

\subsection{A priori estimate I (constant coefficients, principal parts, halfspace)}

We denote by $\mathscr{S}^{\prime}:=\mathscr{S}^{\prime}\left(\mathbb{R}^{n}, \mathbb{C}^{N}\right)$ the space of $\mathbb{C}^{N}$-valued tempered distributions on $\mathbb{R}^{n}$ and by $\mathscr{F}$ the Fourier transform in $\mathscr{S}^{\prime}$.

We put

$$
\begin{array}{rlrl}
\Lambda^{s}(\eta, \xi) & :=\left(|\eta|^{2}+|\xi|^{2}\right)^{s / 2} ; \quad \xi \in \mathbb{R}^{n}, \quad s \in \mathbb{R}, & \eta \in \mathbb{C}, \\
\Lambda^{\sigma}(\eta, \xi):=\operatorname{diag}\left[\Lambda^{\sigma_{1}}(\eta, \xi), \ldots, \Lambda^{\sigma_{r}}(\eta, \xi)\right] ; & \sigma \in \mathbb{R}^{r}, \\
\mathscr{T}^{s}:=\mathscr{F}^{-1} \Lambda^{s}(1, \xi) \mathscr{F} \in \mathscr{L}\left(\mathscr{S}^{\prime}\right) . & &
\end{array}
$$

For $1<p<\infty$ and $s \in \mathbb{R}$ we define the Bessel potential spaces by

$$
H_{p}^{s}:=H_{p}^{s}\left(\mathbb{R}^{n}\right):=\left(\left\{u \in \mathscr{S}^{\prime} \mid \mathscr{T}^{s} u \in L_{p}\left(\mathbb{R}^{n}, \mathbb{C}^{N}\right)\right\},\|\cdot\|_{s, p}\right), \quad\|u\|_{s, p}:=\left\|\mathscr{T}^{s} u\right\|_{p},
$$

and the Besov spaces by real interpolation, that is,

$$
B_{p q}^{s}:=B_{p q}^{s}\left(\mathbb{R}^{n}\right):= \begin{cases}\left(H_{p}^{k}, H_{p}^{k+1}\right)_{s-k, q} & k<s<k+1, \quad k \in \mathbb{Z}, \\ \left(H_{p}^{k-1}, H_{p}^{k+1}\right)_{s / 2, q} & k=s \in \mathbb{Z}\end{cases}
$$

their norms we denote by $\|\cdot\|_{s, p q}$. For further information (interpolation, trace theorems) we refer to $[\mathbf{8}, \mathbf{2 5}]$.

For any $\sigma=\left(\sigma_{1}, \ldots, \sigma_{m N}\right)$ we define the "boundary spaces"

$$
\partial B_{p}^{\alpha}:=\partial B_{p}^{\alpha}\left(\mathbb{R}^{n-1}\right):=\prod_{j=1}^{m N} B_{p p}^{\sigma_{j}-1 / p}\left(\mathbb{R}^{n-1}, \mathbb{C}\right)
$$


and we denote their norm again by $\|\cdot\|_{\sigma, p p}$, without causing confusion. It is not hard to see that $\mathbb{B} \mathbb{R}_{0}(\eta,.) \in C^{\infty}\left(\mathbb{R}^{n-1} \backslash\{0\}, \mathscr{L}\left(\mathbb{C}^{m N}, \mathbb{C}^{m N}\right)\right)$ for all $\eta \in S_{\pi / 4 m}$. Therefore, due to the compactness of $K:=\left\{\left(\eta, \xi^{\prime}\right) \in S_{\pi / 4 m} \times \mathbb{R}^{n-1} \mid \Lambda^{1}\left(\xi^{\prime}, \eta\right)=1\right\}$, the constant

$$
M:=\max _{|\alpha| \leqq[n / 2]+1} \max _{\left(\eta, \xi^{\prime}\right) \in K}\left|\partial_{\xi^{\prime}}^{\alpha}, \mathbb{B} \mathbb{R}_{0}\left(\eta, \xi^{\prime}\right)\right|
$$

is well defined. As a consequence of Lemma 1.5(ii) $\Lambda^{-c}\left(1, \xi^{\prime}\right) \mathbb{B R}_{0}\left(\eta, \xi^{\prime}\right) \Lambda^{b}\left(1, \xi^{\prime}\right)$ is homogeneous of degree 0 and hence by the Michlin-Hörmander multiplier theorem (e.g. $[16,25])$ we can establish the following two lemmas.

LEMma 1.7. For each $\eta \in S_{\pi / 4 m}, \Lambda^{-c}\left(1, \xi^{\prime}\right) \mathbb{B R}_{0}\left(\eta, \xi^{\prime}\right) \Lambda^{b}\left(1, \xi^{\prime}\right)$ is a multiplier in $L_{p}\left(\mathbb{R}^{n-1}, \mathbb{C}^{m N}\right), 1<p<\infty$, and

$$
\left\|\mathscr{F}_{n-1}^{-1} \Lambda^{-c}\left(1, \xi^{\prime}\right) \mathbb{B R}_{0}\left(\eta, \xi^{\prime}\right) \Lambda^{b}\left(1, \xi^{\prime}\right) \mathscr{F}_{n-1}\right\|_{\mathscr{L}\left(L_{p}\left(\mathbb{R}^{n-1}, \mathbb{C}^{m N}\right)\right)} \leqq c(n, N, p, M, \lambda) .
$$

LEMMA 1.8. Let $1<p<\infty$ and $\eta \in S_{\pi / 4 m}$. There exists a constant $\mu_{0}>0$ such that for all $\mu \in\left[\right.$ re $\left.\mu \geqq \mu_{0}\right], \Lambda^{-b}\left(1, \xi^{\prime}\right)\left(\mu+\mathbb{B R}_{0}\left(\eta, \xi^{\prime}\right)\right)^{-1} \Lambda^{c}\left(1, \xi^{\prime}\right)$ is a multiplier in $L_{p}\left(\mathbb{R}^{n-1}, \mathbb{C}^{m N}\right)$ and

$$
\left\|\mathscr{F}_{n-1}^{-1} \Lambda^{-b}\left(1, \xi^{\prime}\right)\left(\mu+\mathbb{B R}_{0}\left(\eta, \xi^{\prime}\right)\right)^{-1} \Lambda^{c}\left(1, \xi^{\prime}\right) \mathscr{F}_{n-1}\right\|_{\mathscr{L}\left(L_{p}\left(\mathbb{R}^{n-1}, \mathbb{C}^{m N}\right)\right)} \leqq c\left(\mu_{0}\right) .
$$

We can now formulate the main result of this section.

TheOREM 1.9. Let $s \in \mathbb{R}, 1<p<\infty, \lambda \in S_{\pi / 2} \backslash\{0\}$. Then there exists a constant $\mu_{0}>0$ such that for each $\mu \in\left[\mathrm{re} \mu \geqq \mu_{0}\right]$ the following two assertions hold:

(i) $\mu+\mathscr{B} \mathscr{R}_{0}(\lambda) \in$ isom $\left(\partial B_{p}^{s-b}\left(\mathbb{R}^{n-1}, \mathbb{C}^{m N}\right), \partial B_{p}^{s-c}\left(\mathbb{R}^{n-1}, \mathbb{C}^{m N}\right)\right)$.

(ii) There exists a constant $c\left(\mu_{0}, M\right)$, such that

$$
\|g\|_{s-b, p p}+|\mu|\|g\|_{s-c, p p} \leqq c\left(\mu_{0}, M\right)\left\|\left(\mu+\mathscr{B} \mathscr{R}_{0}(\lambda)\right) g\right\|_{s-c, p p}
$$

for all $g \in \partial B_{p}^{s-b}\left(\mathbb{R}^{n-1}, \mathbb{C}^{m N}\right)$.

Proof. We choose an arbitrary $g \in \partial B_{p}^{s-c}$. The multiplier theorem and Lemma 1.8 yield

$$
\begin{aligned}
&\left\|\mathscr{F}_{n-1}^{-1}\left(\mu+\mathbb{B R}_{0}\left(\eta, \xi^{\prime}\right)\right)^{-1} \mathscr{F}_{n-1} g\right\|_{s-b, p p} \\
&=\left\|\mathscr{F}_{n-1}^{-1} \Lambda^{-b}\left(1, \xi^{\prime}\right)\left(\mu+\mathbb{B} \mathbb{R}_{0}\left(\eta, \xi^{\prime}\right)\right)^{-1} \Lambda^{c}\left(1, \xi^{\prime}\right) \Lambda^{-c}\left(1, \xi^{\prime}\right) \mathscr{F}_{n-1} g\right\|_{s, p p} \\
& \leqq c\left\|\mathscr{F}_{n-1}^{-1} \Lambda^{-c}\left(1, \xi^{\prime}\right) \mathscr{F}_{n-1} g\right\|_{s, p p} \\
&=c\left\|\mathscr{T}^{-c} g\right\|_{s, p p} \leqq c\|g\|_{s-c, p p}
\end{aligned}
$$

Therefore we have

$$
\left(\mu+\mathscr{B} \mathscr{R}_{0}\right)^{-1} \in \mathscr{L}\left(\partial B_{p}^{s-c}, \partial B_{p}^{s-b}\right) .
$$

Analogously, we find

which proves (i).

$$
\mu+\mathscr{B} \mathscr{R}_{0} \in \mathscr{L}\left(\partial B_{p}^{s-b}, \partial B_{p}^{s-c}\right),
$$

Now let $g \in \partial B_{p}^{s-b}$ be arbitrary. (1.7) implies $\|g\|_{s-b, p p} \leqq c\left\|\left(\mu+\mathscr{B} \mathscr{R}_{0}\right) g\right\|_{s-c, p p}$. Since

$$
\begin{aligned}
\mathbb{B R}_{0}\left(\mu+\mathbb{B R}_{0}\right)^{-1} & =\left(-\mu+\mu+\mathbb{B R}_{0}\right)\left(\mu+\mathbb{B R}_{0}\right)^{-1} \\
& =-\mu\left(\mu+\mathbb{B R}_{0}\right)^{-1}+1
\end{aligned}
$$


we have

$$
\left(\mu+\mathbb{B R} \mathbb{R}_{0}\right)^{-1}=\frac{1}{\mu}\left(1-\mathbb{B R}_{0}\left(\mu+\mathbb{B R}_{0}\right)^{-1}\right)
$$

and so we deduce from (1.7) and (1.8)

Consequently, we see that

$$
\begin{aligned}
\left\|\left(\mu+\mathscr{B} \mathscr{R}_{0}\right)^{-1} g\right\|_{s-c, p p} & =\left\|\frac{1}{\mu}\left(1-\mathscr{B} \mathscr{R}_{0}\left(\mu+\mathscr{R} \mathscr{R}_{0}\right)^{-1}\right) g\right\|_{s-c, p p} \\
& \leqq \frac{1}{|\mu|}\|g\|_{s-c, p p}+\frac{1}{|\mu|} c\|g\|_{s-c, p p} \\
& \leqq \frac{c}{|\mu|}\|g\|_{s-c, p p} .
\end{aligned}
$$

$$
\begin{aligned}
\|g\|_{s-c, p p} & =\left\|\left(\mu+\mathscr{B} \mathscr{R}_{0}\right)^{-1}\left(\mu+\mathscr{B} \mathscr{R}_{0}\right) g\right\|_{s-c, p p} \\
& \leqq \frac{c}{|\mu|}\left\|\left(\mu+\mathscr{B} \mathscr{R}_{0}\right) g\right\|_{s-c, p p},
\end{aligned}
$$

which establishes our assertion.

\subsection{A priori estimate II (variable coefficients, bounded domain, lower terms)}

Our next goal is the extension of Theorem 1.9 to the general situation, that is, we wish to replace the halfspace $\mathbb{H}^{n}$ by a smooth bounded domain in $\mathbb{R}^{n}$. Additionally, we wish to remove the restriction that the operators should have constant coefficients and, finally, the operators should be allowed to have lower order terms. This is done in the usual way via partition of the unity subordinate to coordinate patches and perturbation arguments. For an example of a proof of this kind, see [25]. In our situation, difficulties arise from the fact that we must split up the pseudodifferential operator $\mathscr{B} \mathscr{R}_{0}$ into the Dirichlet problem solution operator $\mathscr{R}_{0}$ and the boundary operator $\mathscr{B}$ again. Now we need some restrictions on $s$, which come from the fact that the trace operator fails to be bounded on $H_{p}^{s}(\Omega)$, if $s \leqq 1 / p$.

Let $\Omega \subset \mathbb{R}^{n}$ be a bounded domain of class $C^{\infty}$. For $s \in \mathbb{R}$ and $1<p<\infty$ we introduce the local Bessel potential and Besov spaces $\left(H_{p}^{s}(\Omega),\|u\|_{s, p, \Omega}\right)$ and $\left(B_{p p}^{s}(\partial \Omega),\|u\|_{s, p p, \partial \Omega}\right)$, respectively, by restrictions (e.g. [25]). The boundary spaces $\partial B_{p}^{\sigma}(\partial \Omega), \quad \sigma=\left(\sigma_{1}, \ldots, \sigma_{m N}\right)$ are defined by $\partial B_{p}^{\sigma}(\partial \Omega):=$ $\prod_{j=1}^{m N} B_{p p}^{\sigma_{j}-1 / p}(\partial \Omega, \mathbb{C})$. We impose the following assumptions: $s \geqq 2 m$ and $1<p<\infty$ are fixed,

$$
\begin{aligned}
& \mathscr{A}(x, D) u(x):=\sum_{|\alpha| \leqq 2 m} a_{\alpha}(x) D^{\alpha} u(x), \\
& a_{\alpha} \in C^{\sigma}\left(\bar{\Omega}, \mathscr{L}\left(\mathbb{C}^{N}, \mathbb{C}^{N}\right)\right), \quad \sigma>s-2 m, \\
& \mathscr{B}^{j}(x, D) u(x):=\sum_{|\beta| \leqq c_{j}} b_{\beta}^{j}(x) D^{\beta} u(x), \quad j=1, \ldots, m N, \\
& b_{\beta}^{j} \in C^{\sigma_{i}}\left(\partial \Omega, \mathscr{L}\left(\mathbb{C}^{N}, \mathbb{C}\right)\right), \quad \sigma_{j}>s-c_{j}-\frac{1}{p}, \quad j=1, \ldots, m N .
\end{aligned}
$$

(A) $\mathscr{A}$ is normally elliptic;

(B) $\mathscr{B}$ satisfies the uniform strong and $\mathscr{B}_{0}$ the normal complementing condition with respect to $\mathscr{A}$. 
In the sequel we will use the following theorem.

THEOREM 1.10. There exist constants $\lambda_{0}>0$ and $c>0$ such that for all $\lambda \in\left[\right.$ re $\left.z \geqq \lambda_{0}\right]$ and $u \in H_{p}^{s}(\Omega)$ the following assertions are true:

(i) $(\lambda+\mathscr{A}(x, D), \mathscr{B}(x, D)) \in$ isom $\left(H_{p}^{s}(\Omega), H_{p}^{s-2 m}(\Omega) \times \partial B_{p}^{s-c}(\partial \Omega)\right)$;

(ii) $\|u\|_{s, p}+|\lambda|\|u\|_{s-2 m, p} \leqq c\left[\left(1+|\lambda|^{(s-2 m) / 2 m}\right)\|(\lambda+\mathscr{A}) u\|_{s-2 m, p}\right.$

$$
\left.+\sum_{k=1}^{m N}\left(1+|\lambda|^{\left(s-c_{k}-1 / p\right) / 2 m}\right)\left\|\mathscr{B}^{j} u\right\|_{s-c_{k}-1 / p, p p}\right] \text {. }
$$

The constants $\lambda_{0}$ and $c$ depend upon the quantities $\left\|a_{\alpha}\right\|_{C^{\sigma}},\left\|b_{\beta}^{j}\right\|_{C^{\sigma}}, m, n, N, p, \Omega$, but not on $\lambda$ and $u$.

Proof. This follows essentially from the $L_{p}$-estimates for elliptic systems (cf. [2] and Agmon's trick [1]; see also [4]).

Because of (B) and Theorem 1.10 , we may assume that $\left(\mathscr{A}, \mathscr{B}_{0}\right) \in$ isom $\left(H_{p}^{s}(\Omega), H_{p}^{s-2 m}(\Omega) \times \partial B_{p}^{s-b}(\partial \Omega)\right)$, adding a positive multiple of the identity to $\mathscr{A}(x, D)$, if necessary. Similarly as above, the operator $\mathscr{R}_{0}$ is given by $\mathscr{R}_{0}(x):=\left(\mathscr{A}(x, D), \mathscr{B}_{0}\right)^{-1}(0, \cdot) \in \mathscr{L}\left(\partial B_{p}^{s-b}(\partial \Omega), H_{p}^{s}(\Omega)\right)$. Finally we need the following hypothesis (which is fulfilled in the case of example (1.2), [15])

(V) There exists a constant $\mu^{*}>0$ such that $\left(\mathscr{A}, \mu \mathscr{B}_{0}+\mathscr{B}\right) \in$ isom $\left(H_{p}^{s}(\Omega)\right.$, $H_{p}^{s-2 m}(\Omega) \times \partial B_{p}^{s-c}(\partial \Omega)$ ) for all $\mu \in\left[\right.$ re $\left.z \geqq \mu^{*}\right]$.

THEOREM 1.11. There are constants $\mu_{0}, c>0$, such that for each $\mu \in\left[\right.$ re $\left.z \geqq \mu_{0}\right]$ the following assertions are true:

(i) $\mu+\mathscr{B R}_{0} \in$ isom $\left(\partial B_{p}^{s-b}(\partial \Omega), \partial B_{p}^{s-c}(\partial \Omega)\right)$,

(ii) $\|g\|_{s-b, p p}+|\mu|\|g\|_{s-c, p p} \leqq c\left\|\left(\mu+\mathscr{B} \mathscr{R}_{0}\right) g\right\|_{s-c, p p}$ for all $g \in \partial B_{p}^{s-b}(\partial \Omega)$.

The constants $\mu_{0}$ and $c$ depend on the quantities $\left\|a_{\alpha}\right\|_{c^{\sigma}},\left\|b_{\beta}^{j}\right\|_{c^{\sigma_{j}}}, m, n, N, p, \Omega$, but not on $\mu$ and $u$.

Proof. See introductory remarks to this section, and [15].

COROLlaRY 1.12. The operator $-\mathscr{B}_{\mathscr{R}_{0}}$ is the generator of an analytic semigroup on $\partial B_{p}^{s-c}(\partial \Omega)$.

Proof. Observe that the domain of the operator $\mathscr{B}_{0}$, namely the space $\partial B_{p}^{s-b}(\partial \Omega)$, is dense in $\partial B_{p}^{s-c}(\partial \Omega)$. In order to show the closedness of $\mathscr{B}_{0}$, consider a sequence $\left(g_{n}\right)_{n \in \mathbb{N}}$ in $\partial B_{p}^{s-b}(\partial \Omega)$, with $g_{n} \rightarrow g$ in $\partial B_{p}^{s-c}(\partial \Omega)$ and $f_{n}:=\mathscr{B} \mathscr{R}_{0} g_{n} \rightarrow f$ in $\partial B_{p}^{s-c}(\partial \Omega)$. Choose $\mu>\mu_{0}$ arbitrarily. Then we have $(\mu+$ $\left.\mathscr{B} \mathscr{R}_{0}\right) g_{n} \rightarrow f+\mu g$ in $\partial B_{p}^{s-c}(\partial \Omega)$, hence $g_{n} \rightarrow\left(\mu+\mathscr{B} \mathscr{R}_{0}\right)^{-1}(f+\mu g) \in \partial B_{p}^{s-b}(\partial \Omega)$. Finally we deduce $\left(\mu+\mathscr{B}_{0}\right)^{-1}(f+\mu g)=g \in \partial B_{p}^{s-b}(\partial \Omega)$ and therefore $\mathscr{B}_{0} g=$ $f$. Now the claim follows from Theorem 1.11 and the characterisation of generators of analytic semigroups (e.g. [20]).

\section{Dynamic boundary value problems}

We now give some illustrations showing how the results of the first part can be applied to (generalisations of) the problems formulated in the introduction. 


\subsection{Problem (P1)}

TheOREM 2.1. The differential operators $\mathscr{A}(x, t, w, D)$ and $\mathscr{B}(x, t, w, D)$ are given by

$$
\begin{gathered}
\mathscr{A}(x, t, w, D) u(x):=\sum_{|\alpha| \leqq 2 m} a_{\alpha}(x, t, w) D^{\alpha} u(x), \\
\mathscr{R}^{j}(x, t, w, D) u(x):=\sum_{|\beta| \leqq c_{j}} b_{\beta}^{j}(x, t, w) D^{\beta} u(x), \quad j=1, \ldots, m N, \quad c \leqq b+m,
\end{gathered}
$$

where $w \in \mathbb{C}^{N}$ and $t \in[0, T]$ for some $T>0$. For every bounded set $W \subset \mathbb{C}^{N}$ the operators $\mathscr{A}, \mathscr{B}$ are supposed to satisfy the hypotheses (A), (B) and (V) uniformly with respect to $(t, w) \in[0, T] \times W$ and, in addition:

$\left(\mathrm{V}^{\prime}\right)$ there exists $s_{0}>0$ such that $\mathscr{R}_{0}(t, w) \in \mathscr{L}\left(\partial B_{p}^{2 m-c}(\partial \Omega), H_{p}^{s_{0}}(\Omega)\right)$ for all $(t, w) \in[0, T] \times \mathbb{C}^{N}$.

We assume without loss of generality $\left(\mathscr{A}(t, w), \mathscr{B}_{0}\right) \in$ isom $\left(H_{p}^{2 m}(\Omega), L_{p}(\Omega) \times\right.$ $\left.\partial B_{p}^{2 m-b}(\partial \Omega)\right)$ for all $(t, w) \in[0, T] \times \mathbb{C}^{N}$. The coefficients are assumed to satisfy (B(., .) stands for bounded mappings)

$$
\begin{aligned}
& \left((t, w) \mapsto a_{\alpha}(., t, w)\right) \in B\left([0, T] \times W, C^{\sigma}\left(\bar{\Omega}, \mathscr{L}\left(\mathbb{C}^{N}\right)\right)\right),|\alpha| \leqq 2 m, \sigma>0 ; \\
& \left((t, w) \mapsto b_{j, \beta}(., t, w)\right) \in B\left([0, T] \times W, C^{\sigma_{j}}\left(\partial \Omega, \mathscr{L}\left(\mathbb{C}^{N}, \mathbb{C}\right)\right)\right),|\beta| \leqq c_{j}, \sigma_{j}>c_{j} ; \\
& \quad j=1, \ldots, m N ; \\
& \left(t \mapsto a_{\alpha}(., t, w)\right) \in C^{\rho}\left([0, T], C^{\sigma}\left(\bar{\Omega}, \mathscr{L}\left(\mathbb{C}^{N}\right)\right)\right),|\alpha| \leqq 2 m ; \\
& \left(t \mapsto b_{j, \beta}(., t, w)\right) \in C^{\rho}\left([0, T], C^{\sigma_{j}}\left(\partial \Omega, \mathscr{L}\left(\mathbb{C}^{N}, \mathbb{C}\right)\right)\right),|\beta| \leqq c_{j}, j=1, \ldots, m N ; \\
& \quad \rho>0,(\text { uniformly with respect to } w \in W) ; \\
& \left(w \mapsto a_{\alpha}(., t, w)\right) \in C^{1-}\left(W, C^{\sigma}\left(\bar{\Omega}, \mathscr{L}\left(\mathbb{C}^{N}\right)\right)\right),|\alpha| \leqq 2 m ; \\
& \left(w \mapsto b_{j, \beta}(., t, w)\right) \in C^{1-}\left(W, C^{\sigma_{j}}\left(\partial \Omega, \mathscr{L}\left(\mathbb{C}^{N}, \mathbb{C}\right)\right)\right),|\beta| \leqq c_{j}, j=1, \ldots, m N ;
\end{aligned}
$$

(uniformly with respect to $t \in[0, T]$ ).

Let $1 \gg \varepsilon>0, p>n /(2 m-\varepsilon)$ and

$$
\begin{aligned}
& F \in C^{0,1-}\left([0, T] \times H^{2 m-\varepsilon}(\Omega), H_{p}^{\varepsilon}(\Omega)\right) \\
& G \in C^{0,1-}\left([0, T] \times \partial B_{p}^{2 m-b-\varepsilon}(\partial \Omega), \partial B_{p}^{2 m-c+\varepsilon}(\partial \Omega)\right) .
\end{aligned}
$$

Then for each initial value $u_{0} \in H_{p}^{2 m}(\Omega)$, the quasilinear problem

$$
\left.\begin{array}{rlrl}
\dot{u}+\mathscr{A}(t, u) u & =F & & \text { in } \Omega \times \mathbb{R}_{+} \\
u)^{-}+\mathscr{B}(t, u) u & =G & & \text { on } \partial \Omega \times \mathbb{R}_{+} \\
u(0) & =u_{0} & & \text { on } \bar{\Omega}
\end{array}\right\}
$$

has an unique maximal solution. The maximal interval of existence $J=J\left(u_{0}\right)$ is right open in $[0, T]$ and $u \in C\left(J, H_{p}^{2 m}(\Omega)\right) \cap C^{1}\left(J, L_{p}(\Omega)\right)$. If $u(t), t \in J$, is bounded in $H_{p}^{2 m-\varepsilon / 2}(\Omega)$ then $u$ is a global solution.

Proof. We verify the hypotheses (Q1) and (Q2) of Amann [5, Theorem 7.1]. As indicated in the introduction, we first decompose the space $H_{p}^{2 m}(\Omega)$. For any $(t, w) \in[0, T] \times W$ we define the operator $A(t, w):=\left.\mathscr{A}(t, w)\right|_{\text {ker } \mathscr{B}}: \operatorname{ker} \mathscr{B} \rightarrow$ $L_{p}(\Omega)$; our assumptions imply $A(t, w) \in$ isom $\left(H_{p, m_{0}}^{2 m}(\Omega), L_{p}(\Omega)\right)$, for all $(t, w) \epsilon$ $[0, T] \times W$, where $H_{p, \mathscr{B}_{0}}^{2 m}(\Omega):=\left\{u \in H_{p}^{2 m}(\Omega) \mid \mathscr{B}_{0} u=0\right\}$. We now fix $(t, w) \epsilon$ $[0, T] \times W$ and omit it in our notation. 
The operator $P:=A^{-1} \mathscr{A} \in \mathscr{L}\left(H_{p}^{2 m}(\Omega), H_{p, \mathscr{R}_{0}}^{2 m}(\Omega)\right)$ clearly satisfies $P^{2}=P$, hence it is a continuous projection and so $H_{p}^{2 m}(\Omega)$ is the topological direct sum $H_{p}^{2 m}(\Omega)=\operatorname{im} P \oplus \operatorname{ker} P=\operatorname{ker} \mathscr{B}_{0} \oplus \operatorname{ker} \mathscr{A}$. We can write any $u \in H_{p}^{2 m}(\Omega)$ in an unique way as

$$
\begin{aligned}
u & =\left(\mathscr{A}, \mathscr{B}_{0}\right)^{-1}\left(\mathscr{A}, \mathscr{B}_{0}\right) u \\
& =\left(\mathscr{A}, \mathscr{B}_{0}\right)^{-1}(\mathscr{A} u, 0)+\left(\mathscr{A}, \mathscr{B}_{0}\right)^{-1}\left(0, \mathscr{B}_{0} u\right) \\
& =\left(\mathscr{A}, \mathscr{B}_{0}\right)^{-1}(\mathscr{A} u, 0)+\mathscr{R}_{0} \mathscr{B}_{0} u \in \operatorname{ker} \mathscr{B}_{0}+\operatorname{ker} \mathscr{A}
\end{aligned}
$$

and we first consider the system

$$
\left.\begin{array}{rlrl}
\dot{u}+\mathscr{A}(t, w)\left(u-\mathscr{R}_{0}(t, w) \mathscr{B}_{0} u\right) & =F(t, u) & & \text { in } \Omega \times \mathbb{R}_{+}, \\
\left(\mathscr{B}_{0} u\right)^{\circ}+\mathscr{B}(t, w) u & =G(t, u)) & & \text { on } \partial \Omega \times \mathbb{R}_{+}, \\
u(0) & =u_{0} & & \text { on } \bar{\Omega},
\end{array}\right\}
$$

instead of (P1).

Since $\mathscr{B}_{0} \in \mathscr{L}\left(H_{p}^{2 m}(\Omega), \partial B_{p}^{2 m-b}(\Omega)\right), E_{1}:=\operatorname{graph}\left(\mathscr{B}_{0}\right)$ is a closed subspace of $H_{p}^{2 m} \times \partial B_{p}^{2 m-b}(\partial \Omega)$ and so a Banach space with the induced topology.

We define the unbounded linear operator

$$
\begin{aligned}
\mathbb{A}(t, w): L_{p} \times \partial B_{p}^{2 m-c} \supset \operatorname{graph}\left(\mathscr{B}_{0}\right) & \rightarrow L_{p} \times \partial B_{p}^{2 m-c} \\
(u, z) & \mapsto\left(\mathscr{A}(t, w)\left(u-\mathscr{R}_{0}(t, w) z\right), \mathscr{B}(t, w) u\right)
\end{aligned}
$$

and rewrite $(*)$ as

$$
\begin{aligned}
\dot{v}+\mathbb{A}(t, w) v & =H(v), \quad H:=(F, G), \quad t>0, \\
v(0) & =v_{0},
\end{aligned}
$$

where $v:=(u, z)$.

The domain $E_{1}$ of the operator $A$ is somewhat unusual. To fit our situation into the framework of [6], we need some preparatory considerations. Since $c \leqq b+m$, there exists a right inverse $\mathscr{C} \in \mathscr{L}\left(\partial B_{p}^{2 m-c}(\Omega), L_{p}(\Omega)\right) \cap \mathscr{L}\left(\partial B_{p}^{2 m-b}(\Omega), H_{p}^{2 m}(\Omega)\right)$ of the Dirichlet operator. We now introduce the spaces

$$
\begin{aligned}
E_{0} & :=L_{p}(\Omega) \times \partial B_{p}^{2 m-c}(\partial \Omega), \\
E^{*} & :=H_{p, \mathscr{B}_{0}}^{2 m}(\Omega) \times \partial B_{p}^{2 m-b}(\partial \Omega), \\
\tilde{E} & :=H_{p}^{2 m}(\Omega) \times \partial B_{p}^{2 m-b}(\partial \Omega)
\end{aligned}
$$

and, by complex interpolation $[., .]_{\theta}, 0 \leqq \theta \leqq 1$, the spaces

$$
\begin{aligned}
& E_{\theta}:=\left[E_{0}, E_{1}\right]_{\theta}, \\
& E_{\theta}^{*}:=\left[E_{0}, E^{*}\right]_{\theta}, \\
& \tilde{E}_{\theta}:=\left[E_{0}, \tilde{E}\right]_{\theta} .
\end{aligned}
$$

The map $\varphi:(u, z) \mapsto(u+\mathscr{b} z, z)$ satisfies

and therefore

$$
\varphi \in \operatorname{isom}\left(E^{*}, E_{1}\right) \cap \text { aut }(\tilde{E}) \cap \text { aut }\left(E_{0}\right),
$$

Indeed, the estimates

$$
\varphi \in \text { aut }\left(\widetilde{E}_{\theta}\right), \quad 0 \leqq \theta \leqq 1 .
$$

$$
\begin{aligned}
\|(u+\mathscr{C} z, z)\|_{\tilde{E}} & \leqq u\left\|_{2 m, p}+\right\| \mathscr{C} z\left\|_{2 m, p}+\right\| z \|_{2 m-b, p p} \\
& \leqq\|u\|_{2 m, p}+c\|z\|_{2 m-b, p p}
\end{aligned}
$$


hold, and an inverse $\varphi^{-1}$ is given by $(u, z) \mapsto(u-\mathscr{C} z, z)$, which proves $\varphi \in$ aut $(\tilde{E})$. The rest of $(2.1)$ follows similarly, and (2.2) is a consequence of interpolation properties. With a result of Seeley [27], it can be shown [4] that $\left[L_{p}, H_{p, P_{0}}^{2 m}\right]_{\theta}=H_{p, Q_{0}}^{2 m \theta}$ for all values of $0<\theta<1, k \neq 2 m \theta-1 / p, k=0, \ldots, m-1$, where $H_{p, m_{0}}^{s}=H_{p}^{s}$, provided $0 \leqq s \leqq 1 / p$. Consequently, we have $E_{\theta}^{*}=\tilde{E}_{\theta}$, provided $0<\theta<1 / 2 m p$.

Due to (2.1) and the properties of the complex interpolation functor, $\varphi$ induces an isomorphism of the spaces $E_{\theta} \cong E_{\theta}^{r *}, 0 \leqq \theta \leqq 1$, and with (2.2) we see that $E_{\theta}=\tilde{E}_{\theta}$ for all values of $0<\theta<1 / 2 \mathrm{mp}$.

We deduce from the considerations above that by a suitable choice of $0<\gamma<\beta<\alpha<1$ we can find a chain of inclusions $E_{1} \subset E_{\alpha} \subset E_{\beta} \subset H_{p}^{2 m-\varepsilon}(\Omega) \times$ $\partial B_{p}^{2 m-\varepsilon-b}(\partial \Omega) \subset H_{p}^{\varepsilon}(\Omega) \times \partial B_{p}^{2 m+\varepsilon-c}(\partial \Omega) \subset E_{\gamma} \subset E_{0}$ which proves [6, (Q1)].

Now we show $(\mathrm{Q} 2)$. First we prove $((t, w) \mapsto \mathbb{A}(t, w)) \in C_{T}^{\sigma}\left(\mathscr{H}\left(E_{0}, E_{1}\right)\right)$, that is, $\{A(t, w),(t, w) \in[0, T] \times B\} \subset \mathscr{H}\left(E_{0}, E_{1}\right)$ is a regularly bounded family of generators of analytic semigroups in $E_{0}$ with domain $E_{1}$.

Let $(t, w) \in[0, T] \times B$ be fixed for a moment. Due to Theorems 1.10 and 1.11 there exists a constant $\lambda_{0}$ such that

$$
\begin{aligned}
\left(\lambda+\mathscr{A}, \mathscr{B}_{0}\right) & \in \text { isom }\left(H_{p}^{2 m}(\Omega), L_{p}(\Omega) \times \partial B_{p}^{2 m-b}(\partial \Omega)\right), \\
\left(\lambda+\mathscr{B} \mathscr{R}_{0}\right) & \in \text { isom }\left(B_{p}^{2 m-b}(\partial \Omega), B_{p}^{2 m-c}(\partial \Omega)\right),
\end{aligned}
$$

and, in particular, $\lambda+A \in$ isom $\left(H_{p, g_{0}}^{2 m}(\Omega), L_{p}(\Omega)\right)$ for all values of $\lambda \in$ [re $\lambda \geqq \lambda_{0}$ ]. Let $(x, y) \in E_{0}$, re $\lambda \geqq \lambda_{0}$, and $(u, z) \in$ graph $\left(\mathscr{B}_{0}\right)$ satisfy the equations

$$
\begin{aligned}
\lambda u+\mathscr{A}\left(u-\mathscr{R}_{0} z\right) & =x, \\
\lambda z+\mathscr{B} u & =y .
\end{aligned}
$$

Then

$$
\begin{aligned}
(2.3) & \Rightarrow \lambda\left(u-\mathscr{R}_{0} z\right)+A\left(u-\mathscr{R}_{0} z\right)=x-\lambda \mathscr{R}_{0} z \\
& \Rightarrow u-\mathscr{R}_{0} z=(\lambda+A)^{-1} x-\lambda(\lambda+A)^{-1} \mathscr{R}_{0} z \\
& \Rightarrow u=(\lambda+A)^{-1} x+\left(1-\lambda(\lambda+A)^{-1}\right) \mathscr{R}_{0} z
\end{aligned}
$$

and

$$
\begin{aligned}
(2.4) \Rightarrow & \lambda z+\mathscr{B}_{0} z+\mathscr{B}\left(u-\mathscr{R}_{0} z\right)=y \\
\Rightarrow z= & \left(\lambda+\mathscr{B} \mathscr{R}_{0}\right)^{-1} y-\left(\lambda+\mathscr{B} \mathscr{R}_{0}\right)^{-1} \mathscr{B}\left(u-\mathscr{R}_{0} z\right), \\
(2.5) \Rightarrow z= & \left(\lambda+\mathscr{B} \mathscr{R}_{0}\right)^{-1} y-\left(\lambda+\mathscr{B} \mathscr{R}_{0}\right)^{-1} \mathscr{B}(\lambda+A)^{-1} x \\
& +\left(\lambda+\mathscr{B} \mathscr{R}_{0}\right)^{-1} \lambda \mathscr{B}(\lambda+A)^{-1} \mathscr{R}_{0} z .
\end{aligned}
$$

These facts imply

$$
\left(1-\lambda\left(\lambda+\mathscr{B} \mathscr{R}_{0}\right)^{-1} \mathscr{B}(\lambda+A)^{-1} \mathscr{R}_{0}\right) z=\left(\lambda+\mathscr{B} \mathscr{R}_{0}\right)^{-1}\left(y-\mathscr{B}(\lambda+A)^{-1} x\right) .
$$

Without loss of generality, we may assume $s_{0}<1 / p$; therefore we have $H_{p, \mathscr{S}_{0}}^{s_{0}}=H_{p}^{s_{0}}$. By interpolating the estimates $\left\|(\lambda+A)^{-1}\right\|_{\mathscr{L}\left(H_{p, w_{0}}^{2 m}\right)} \leqq c /|\lambda|[6]$ and $\left\|(\lambda+A)^{-1}\right\|_{\mathscr{L}\left(L_{p}, H_{p}^{2 m}\right)} \leqq c$ (Theorem 1.10), it follows that $\left\|(\lambda+A)^{-1}\right\|_{L\left(H_{p}^{s_{0}}, H_{p}^{2 m}\right)} \leqq$ $c /|\lambda|^{s_{0} / 2 m}$. 
Now, by Theorem 1.10 we can conclude that

$$
\begin{aligned}
\left\|\lambda\left(\lambda+\mathscr{B} \mathscr{R}_{0}\right)^{-1} \mathscr{B}(\lambda+A)^{-1} \mathscr{R}_{0} z\right\|_{2 m-c, p p, \partial \Omega} & \leqq\left\|(\lambda+A)^{-1} \mathscr{R}_{0} z\right\|_{2 m, p, \Omega} \\
& \leqq \frac{c}{|\lambda|^{s_{0} / 2 m}}\left\|\mathscr{R}_{0} z\right\|_{s_{0}, p, \Omega} \\
& \left.\leqq \frac{c}{|\lambda|^{s_{\delta} / 2 m}}\|z\|_{2 m-c, p p, \partial \Omega} \text { (due to }\left(\mathrm{V}^{\prime}\right)\right),
\end{aligned}
$$

so that, for sufficiently large $\lambda, \quad\left(1-\lambda\left(\lambda+\mathscr{B} \mathscr{R}_{0}\right)^{-1} \mathscr{B}(\lambda+A)^{-1} \mathscr{R}_{0}\right) \epsilon$ aut $\left(\partial B_{p}^{2 m-c}(\partial \Omega)\right)$, and there exists a constant $\lambda_{1} \geqq 0$ such that the norm of this operator is bounded by $\frac{1}{2}$ independently of $|\lambda| \geqq \lambda_{1}$.

Theorems 1.10 and 1.11, together wtih (2.7), imply

$$
\|z\|_{2 m-c, p p, \partial \Omega} \leqq \frac{c}{|\lambda|}\left(\|y\|_{2 m-c, p p, \partial \Omega}+\|x\|_{p, \Omega}\right) .
$$

In addition, from (2.6) we deduce the estimate

$$
\begin{aligned}
\|u\|_{p, \Omega} & \leqq \frac{c}{|\lambda|}\|x\|_{p, \Omega}+\left\|\left(1-\lambda(\lambda+A)^{-1}\right) \mathscr{R}_{0} z\right\|_{p, \Omega} \\
& \leqq c\left(\frac{1}{|\lambda|}\|x\|_{p, \Omega}+\left\|\mathscr{R}_{0} z\right\|_{p, \Omega}\right) \\
& \leqq c\left(\frac{1}{|\lambda|}\|x\|_{p, \Omega}+\|z\|_{2 m-c, p p, \partial \Omega}\right) \quad\left(\operatorname{see}\left(\mathrm{V}^{\prime}\right)\right) \\
& \leqq \frac{c}{|\lambda|}\left(\|x\|_{p, \Omega}+\|y\|_{2 m-c, p p, \Omega}\right) .
\end{aligned}
$$

Finally, making $\lambda_{0}$ bigger, if necessary, (2.8) and (2.9) yield

$$
\left\|(\lambda+\mathcal{A})^{-1}(x, y)\right\|_{L_{p}(\Omega) \times \partial B_{p}^{2 m-c}} \leqq \frac{c}{|\lambda|}\|(x, y)\|_{L_{p}(\Omega) \times \partial B_{p}^{2 m-c}}
$$

for all $\lambda \in\left[\right.$ re $\left.\lambda \geqq \lambda_{0}\right]$. Obviously $\mathbb{A}$ is densely defined. The proof of its closedness is easy (see Corollary 1.12).

Since the function $(t, w) \mapsto\left(c(t, w), \lambda_{0}(t, w)\right)$ is bounded on bounded sets by Theorems 1.10 and 1.11 , it follows that $\{\mathbb{A}(t, w),(t, w) \in[0, T] \times W\} \subset$ $\mathscr{H}\left(E_{0}, E_{1}\right)$ is regularly bounded. From our hypotheses on the coefficients, it follows easily that $\mathbb{A}(., w) \in C^{\rho}\left([0, T), \mathscr{L}\left(E_{1}, E_{0}\right)\right)$, uniformly with respect to $w \in W$.

Finally, it remains to show that $((t, w) \mapsto \mathbb{A}(t, w)) \in C^{\rho, 1-}([0, T] \times$ $\left.E_{\beta}, \mathscr{H}\left(E_{1}, E_{0}\right)\right)$. Because of $p>n /(2 m-\varepsilon)$ and the Sobolev embedding theorem, $H_{p}^{2 m-\varepsilon}(\Omega) \hookrightarrow C(\bar{\Omega})$, we have $a_{\alpha}(x, t,),. b_{\beta}^{j}(x, t,.) \in C^{1-}\left(E_{\beta}\right)$ for all values of $|\alpha| \leqq 2 m,|\beta| \leqq c_{j}, j=1, \ldots, m N$, uniformly with respect to $(x, t) \in \overline{\mathbf{\Omega}} \times[0, T]$. Thus the hypotheses of [6, Theorem 7.1] are fulfilled and so

$$
\begin{aligned}
\dot{v}+\mathbb{A}(t, v) v & =H(v), \quad t>0, \\
v(0) & =v_{0}
\end{aligned}
$$

possesses for each $v_{0} \in \operatorname{graph}\left(\mathscr{B}_{0}\right)$ exactly one maximal solution. The stated 
regularity of the solution at $t=0$ follows from [6, Theorem (5.3)]. Recalling the definition of $A$, the assertions follow.

Corollary 2.2. Let $m=N=1, p>n, \mathscr{A}=-\Delta, \mathscr{B}=\partial / \partial v, f, g \in C^{2-}(\mathbb{R})$. By $F$ and $G$ we denote the substitution (Nemitskii) operators induced by $f$ and $g$, respectively. Then problem (P1) has for each $u_{0} \in H_{p}^{2}(\Omega)$ a unique maximal solution $u \in C\left(J, H_{p}^{2}(\Omega)\right) \cap C^{1}\left(J, L_{p}(\Omega)\right)$. If $u(t), t \in J$, is bounded in $H_{p}^{2-\varepsilon}(\Omega)$, the solution is global.

Proof. It is implied by [4, Proposition (15.4)] that $F \in C^{1-}\left(H_{p}^{2 m-\varepsilon}(\Omega), H_{p}^{\varepsilon}(\Omega)\right)$ and $G \in C^{1-}\left(B_{p p}^{1-(1 / p)-\varepsilon}(\partial \Omega), B_{p p}^{2-(1 / p)+\varepsilon}(\partial \Omega)\right)$ for a suitable $\varepsilon>0$. Now the assertion follows from Theorem 2.1.

\subsection{Problem (P2)}

THEOREM 2.3. Let $s \geqq 2 m$. The operators $\mathscr{A}$ and $\mathscr{B}$ satisfy the hypotheses (A), (B) and (V). Moreover, we are given $0<\varepsilon \ll 1$ and functions $f \in$ $C^{1}\left(\mathbb{R}_{+}, H_{p}^{s-2 m-\varepsilon}(\Omega)\right)$ and $G \in C^{1-}\left(\partial B_{p}^{s-b-\varepsilon}(\partial \Omega), \partial B_{p}^{s-c+\varepsilon}(\partial \Omega)\right)$. Then the semilinear problem

$$
\left.\begin{array}{rlrl}
\mathscr{A}(x, D) u & =f & & \text { in } \Omega \times \mathbb{R}_{+}, \\
\dot{u}+\mathscr{B}(x, D) u & =G(u) & & \text { on } \partial \Omega \times \mathbb{R}_{+}, \\
u(0) & =z_{0} & & \text { on } \partial \Omega,
\end{array}\right\}
$$

possesses for each initial value $z_{0} \in \partial B_{p}^{s-b}(\partial \Omega)$ a unique maximal solution $u \in C\left(\left[0, t^{+}\right), H_{p}^{s}(\Omega)\right) \cap C^{1}\left(\left(0, t^{+}\right), H_{p}^{s-m}(\Omega)\right), t^{+}>0$.

Proof. Letting $\mathscr{B}_{0} u:=z$, (P2) is equivalent to the problem $\mathscr{A}\left(u-\mathscr{R}_{0} z\right)=f$ in $\Omega \times \mathbb{R}_{+}$,

$$
\left.\begin{array}{rlrl}
\dot{z}+\mathscr{B} \mathscr{R}_{0} z & =G-\mathscr{B}\left(u-\mathscr{R}_{0} z\right)=G-\mathscr{B}\left(\mathscr{A}, \mathscr{B}_{0}\right)^{-1}(f, 0) & & \text { on } \partial \Omega \times \mathbb{R}_{+}, \\
z(0) & =z_{0} & & \text { on } \partial \Omega .
\end{array}\right\}
$$

The assumptions imply $G-\mathscr{B}\left(\mathscr{A}, \mathscr{B}_{0}\right)^{-1}(f, 0) \in C^{0.1-}\left(\mathbb{R}_{+} \times \partial B_{p}^{s-b-\varepsilon}(\partial \Omega)\right.$, $\left.\partial B_{p}^{s-c+\varepsilon}(\partial \Omega)\right)$. For each $z_{0} \in \partial B_{p}^{s-b}(\partial \Omega)$, [6, Theorem (7.1)] guarantees the existence of a unique maximal solution $z \in C\left(\left[0, t^{+}\right), \partial B_{p}^{s-b}(\partial \Omega)\right) \cap C^{1}\left(\left(0, t^{+}\right)\right.$, $\left.\partial B_{p}^{s-c}(\partial \Omega)\right)$ of the Cauchy problem $\left({ }^{*}\right)$. Hence $u:=\left(\mathscr{A}, \mathscr{B}_{0}\right)^{-1}(f, z)$ satisfies the assertion.

Corollary 2.4. Let $m=N=1, p>n, f \in C^{1}\left(\mathbb{R}_{+}, H^{-\varepsilon}(\Omega)\right)$ and $g \in C^{1}(\mathbb{R})$. By $G$ we denote the substitution operator induced by $g$. Then for each initial value $z_{0} \in B_{p p}^{2-(1 / p)}(\partial \Omega)$, the problem

$$
\begin{aligned}
-\Delta u & =f & & \text { in } \Omega \times \mathbb{R}_{+}, \\
\dot{u}+\frac{\partial}{\partial v} u & =G(u) & & \text { on } \partial \Omega \times \mathbb{R}_{+}, \\
u(0) & =z_{0} & & \text { on } \partial \Omega,
\end{aligned}
$$

has a unique maximal solution $u \in C\left(\left[0, t^{+}\right), H_{p}^{2}(\Omega)\right) \cap C^{1}\left(\left(0, t^{+}\right), H_{p}^{1}(\Omega)\right)$.

Proof. It is implied by [4, Proposition (15.4)] that the Nemitskii operator $G$ satisfies the hypotheses of Theorem 2.3. Since the constraints (A), (B) and (V) are easily verified, the assertion follows from Theorem 2.3 . 


\subsection{Problem (P3)}

In this section, we occupy ourselves with the problem

$$
\left.\begin{array}{rl}
-\Delta u=f & \text { in } \Omega \times \mathbb{R}, \\
+\frac{\partial}{\partial v} u=g & \text { on } \partial \Omega \times \mathbb{R}, \\
u(0)=z_{0} & \text { on } \partial \Omega, \\
\dot{u}(0)=z_{1} & \text { on } \partial \Omega .
\end{array}\right\}
$$

First of all we study the linear case. We are given an open interval $J$ containing zero and functions $f \in C\left(J, L_{2}(\Omega)\right)$ and $g \in C\left(J, L_{2}(\partial \Omega)\right)$. We put $\mathscr{A}:=-\Delta$, $\mathscr{B}:=\partial / \partial v, \mathscr{R}_{0}:=\left(\mathscr{A}, \mathscr{B}_{0}\right)^{-1}(0,), z:.=\mathscr{B}_{0} u$. Then (P3) is equivalent to

$$
\left.\begin{array}{rlrl}
\mathscr{A}\left(u-\mathscr{R}_{0} z\right) & =f & & \text { in } \Omega \times J, \\
\ddot{z}+\mathscr{B}_{0} z & =g-\mathscr{B}\left(\mathscr{A}, \mathscr{B}_{0}\right)^{-1}(f, 0) & & \text { on } \partial \Omega \times J, \\
z(0) & =z_{0} & & \text { on } \partial \Omega, \\
\dot{z}(0) & =z_{1} & & \text { on } \partial \Omega .
\end{array}\right\}
$$

Hence it is useful to study the operator $\mathscr{B} \mathscr{R}_{0}$ first.

We identify the dual space of $L_{2}(\partial \Omega)$ with $L_{2}(\partial \Omega)$ and denote by $\langle\cdot, \cdot\rangle_{\partial \Omega}$ the duality pairing in $L_{2}(\partial \Omega)$. Let $g, h \in H^{\frac{1}{2}}(\partial \Omega)$, where $H^{s}:=H_{2}^{s}:=B_{22}^{s}$. Gauss' theorem yields the estimate

$$
\begin{aligned}
\left|\left\langle\mathscr{B} \mathscr{R}_{0} g, h\right\rangle_{\partial \Omega}\right|=\left|\int_{\Omega} \nabla \mathscr{R}_{0} g \nabla \mathscr{R}_{0} h d x\right| & \leqq\left\|\mathscr{R}_{0} g\right\|_{1,2, \Omega}\left\|\mathscr{R}_{0} h\right\|_{1,2, \Omega} \\
& \leqq c\|g\|_{\frac{1}{2}, 2, \partial \Omega}\|h\|_{\frac{1}{2}, 2, \partial \Omega},
\end{aligned}
$$

and so $\mathscr{B} \mathscr{R}_{0} \in \mathscr{L}\left(H^{\frac{1}{2}}(\partial \Omega), H^{-\frac{1}{2}}(\partial \Omega)\right)$. Because of Theorem 1.11 , there exists a constant $\mu>0$ such that $\mu+\mathscr{B} \mathscr{R}_{0} \in$ isom $\left(H^{\frac{3}{2}}(\partial \Omega), H^{\frac{1}{2}}(\partial \Omega)\right)$; hence, by interpolation,

$$
\mu+\mathscr{B} \mathscr{R}_{0} \in \mathscr{L}\left(H^{1}(\partial \Omega), L_{2}(\partial \Omega)\right) .
$$

Denoting the inner product in $L_{2}(\partial \Omega)$ by $(\cdot, \cdot)_{\partial}$, it follows for all $g \in H^{1}(\partial \Omega)$, by Poincaré's inequality, that

$$
\left(\mu+\mathscr{B} \mathscr{R}_{0} g, g\right)_{\partial}=\int_{\Omega}\left|\nabla \mathscr{R}_{0} g\right|^{2} d x+\int_{\partial \Omega} \mu|g|^{2} d \sigma \geqq c\left\|\mathscr{R}_{0} g\right\|_{1,2, \Omega}^{2} \geqq c\|g\|_{0,2, \partial \Omega}^{2} .
$$

Therefore $\mu+\mathscr{B} \mathscr{R}_{0}$ is positive, symmetric, injective and has closed range. From (2.12) it follows that $R\left(\mu+\mathscr{B} \mathscr{R}_{0}\right)=L_{2}$ and so $\mu+\mathscr{B} \mathscr{R}_{0}$ is self-adjoint. Hence $\mathscr{B} \mathscr{R}_{0}$ generates a $C^{0}$-semigroup on $L_{2}$ and we can apply the results of [5, Theorem 6 of the appendix] on semigroups in interpolation and extrapolation spaces. In particular, for each $\beta>0$ the $H^{\beta}(\partial \Omega)$-realisation $\left(\mathscr{B} \mathscr{R}_{0}\right)_{\beta}$ of $\mathscr{B} \mathscr{R}_{0}$ has domain $H^{\beta+1}(\partial \Omega)$ and generates a $C^{0}$-semigroup on $H^{\beta}(\partial \Omega)$.

We now rewrite the second order equation (2.11) as the first order system

$$
\left[\begin{array}{c}
z(t) \\
\dot{z}(t)
\end{array}\right]^{\cdot}+\left[\begin{array}{cr}
0 & -1 \\
\mathscr{B} \mathscr{R}_{0} & 0
\end{array}\right]\left[\begin{array}{c}
z(t) \\
\dot{z}(t)
\end{array}\right]=\left[\begin{array}{c}
0 \\
g(t)-\mathscr{B}\left(\mathscr{A}, \mathscr{B}_{0}\right)^{-1}(f(t), 0)
\end{array}\right], \quad t \in J
$$


The operator

$$
A:=\left[\begin{array}{cr}
0 & -1 \\
\mathscr{B} \mathscr{R}_{0} & 0
\end{array}\right]
$$

generates a $C_{0}$-semigroup on $H^{\frac{1}{2}}(\partial \Omega) \times L_{2}(\partial \Omega)$, and we have the corresponding results in the interpolation spaces $H^{\beta+\frac{1}{2}}(\partial \Omega) \times H^{\beta}(\partial \Omega), \beta \geqq 0$.

In the following, let $s \geqq 1$. Given functions $f \in C\left(J, H^{s-1}(\Omega)\right)$ and $g \in$ $C\left(J, H^{s-\frac{1}{2}}(\partial \Omega)\right)$, any function $u \in C\left(J, H^{s+1}(\Omega)\right)$ with trace $z:=\mathscr{B}_{0} u \in$ $C^{1}\left(J, H^{s}(\partial \Omega)\right)$ on the boundary, which satisfies the equations

$$
u(t)=\left(\mathscr{A}, \mathscr{B}_{0}\right)^{-1}(f(t), z),
$$

$$
\left[\begin{array}{c}
z(t) \\
\dot{z}(t)
\end{array}\right]=e^{-t \mathbb{A}}\left[\begin{array}{c}
z(0) \\
\dot{z}(0)
\end{array}\right]+\int_{0}^{t} e^{-(t-\tau) \mathbb{A}}\left[\begin{array}{c}
0 \\
g(\tau)-\mathscr{B}\left(\mathscr{A}, \mathscr{B}_{0}\right)^{-1}(f(\tau), 0)
\end{array}\right] d \tau, \quad t \in J
$$

is called a mild solution of the problem (P3). A mild solution of (P3) need not be a solution in the classical sense. Therefore we introduce the notion of weak solutions. (Although the relation between mild solutions and weak solutions for abstract linear evolution equations is well known, those results do not apply to our situation, since it is not obvious how the operators appearing in the formula for the mild solution are related to the original differential equation, that is, to the terms appearing in the standard formulation of a weak solution for our problem.)

Given $f \in C\left(J, L_{2}(\Omega)\right)$ and $g \in C\left(J, L_{2}(\partial \Omega)\right)$, any function $u \in C\left(J, H_{2}^{1}(\Omega)\right)$ with trace $z:=\mathscr{B}_{0} u \in C^{1}\left(J, L_{2}(\partial \Omega)\right)$ is said to be a weak solution of problem (P3), provided

$$
\begin{array}{r}
\int_{0}^{T}\left[\langle\nabla \varphi(t), \nabla u(t)\rangle_{\partial \Omega}-\langle\dot{\varphi}(t), \dot{z}(t)\rangle_{\partial \Omega} d t+\langle\varphi(T), \dot{z}(T)\rangle_{\partial \Omega}-\langle\varphi(0), \dot{z}(0)\rangle_{\partial \Omega}\right. \\
=\int_{0}^{T}\left[\langle\varphi(t), f(t)\rangle_{\partial \Omega}+\langle\varphi(t), g(t)\rangle_{\partial \Omega}\right] d t \forall \varphi \in C\left(J, H^{1}(\Omega)\right) \cap C^{1}\left(J, H^{\frac{1}{2}+\varepsilon}(\Omega)\right), \\
\varepsilon>0, \quad T \in J .
\end{array}
$$

This formula is obtained by multiplication (in the sense of the duality pairing) of equations (P3) by $\varphi$, addition of the equations, integration over time and partial integration.

Lemma 2.5. Suppose that $f \in C\left(J, H^{s-1}(\Omega)\right), \quad g \in C\left(J, H^{s-\frac{1}{2}}(\partial \Omega)\right), \quad\left(z_{0}, z_{1}\right) \epsilon$ $H^{s+\frac{1}{2}}(\partial \Omega) \times H^{s}(\partial \Omega)$, and that the function $(z, v)$, defined by

satisfies

$$
t \mapsto\left[\begin{array}{c}
z(t) \\
v(t)
\end{array}\right]=e^{-t \mathbb{A}}\left[\begin{array}{c}
z_{0} \\
z_{1}
\end{array}\right]+\int_{0}^{t} e^{-(t-\tau) \mathbb{A}}\left[\begin{array}{c}
0 \\
g(\tau)-\mathscr{B}\left(\mathscr{A}, \mathscr{B}_{0}\right)^{-1}(f(\tau), 0)
\end{array}\right] d \tau,
$$

$$
(z, v) \in C\left(J, H^{s+\frac{1}{2}}(\partial \Omega)\right) \times C\left(J, H^{s}(\partial \Omega)\right) .
$$

Then the following assertions are true:

(i) $z \in C^{1}\left(J, H^{s}(\partial \Omega)\right)$ and $\dot{z}=v$, which means that $u:=\left(\mathscr{A}, \mathscr{B}_{0}\right)^{-1}(f, z)$ is a mild solution of (P3).

(ii) $u$ is a weak solution of (P3).

(iii) Any weak solution $u \in C\left(J, H^{s+1}(\Omega)\right)$ with trace $z:=\mathscr{B}_{0} u \in C^{1}\left(J, H^{s}(\partial \Omega)\right)$ on $\partial \Omega$ of $(\mathrm{P} 3)$ is a mild solution, provided the regularity assumptions on $f$ and $g$ above hold. 
Proof. For the lengthy but straightforward proof we refer to [15].

THEOREM 2.6. Let $s \geqq 1, f \in C\left(\mathbb{R}, H^{s-1}(\Omega)\right)$ and $G \in C^{1-}\left(H^{s+\frac{1}{2}}(\partial \Omega)\right.$, $\left.H^{s-\frac{1}{2}}(\partial \Omega)\right)$, and suppose that $f$ and $G$ are bounded on bounded sets. Then for each initial value $\left(z_{0}, z_{1}\right) \in H^{s+\frac{1}{2}}(\partial \Omega) \times H^{s}(\partial \Omega)$ there exists a maximal open interval $J=\left(T^{-}, T^{+}\right)$with $0 \in J$ and an unique weak solution $u \in C\left[\left(0, T^{+}\right), H^{s+1}(\Omega)\right)$ with trace $z:=\mathscr{B}_{0} u \in C^{1}\left(\left[0, T^{+}\right), H^{s}(\partial \Omega)\right)$ of the semilinear problem

$$
\begin{aligned}
-\Delta u & =f & & \text { in } \Omega \times \mathbb{R}, \\
+\frac{\partial}{\partial v} u & =G(u) & & \text { on } \partial \Omega \times \mathbb{R}, \\
u(0) & =z_{0} & & \text { on } \partial \Omega, \\
\dot{u}(0) & =z_{1} & & \text { on } \partial \Omega .
\end{aligned}
$$

If there exists a function $k \in C(\mathbb{R}, \mathbb{R})$ such that $\|z(t)\|_{s+\frac{1}{2,2, a \Omega}}+\|\dot{z}(t)\|_{s, 2, a \Omega} \leqq k(t)$ for all $t \in\left[0, T^{+}\right)$, then $T^{+}=\infty$ (analogous result for $\left.T^{-}\right)$.

Proof. Banach iteration and usual continuation arguments (e.g. Pazy [20, Chapter 6]) yield the interval $J$ and a unique solution $(z, v) \in C\left(J, H^{s+\frac{1}{2}}(\partial \Omega) \times\right.$ $H^{s}(\partial \Omega)$ ) of the equation

$$
\left[\begin{array}{l}
z(t) \\
v(t)
\end{array}\right]=e^{-t \mathbb{A}}\left[\begin{array}{l}
z_{0} \\
z_{1}
\end{array}\right]+\int_{0}^{t} e^{-(t-\tau) \mathbb{A}}\left[\begin{array}{c}
0 \\
G(z(\tau))-\mathscr{B}\left(\mathscr{A}, \mathscr{B}_{0}\right)^{-1}(f(\tau), 0)
\end{array}\right] d \tau
$$

such that

$$
\lim _{t \rightarrow T^{+}}\left(\|z(t)\|_{s+\frac{1}{2}, 2, \partial \Omega}+\|v(t)\|_{s, 2, \partial \Omega}\right)=\infty,
$$

if $T^{+}<\infty$. We put $g(t):=G(z(t)), t \in J$. Now the assertion follows from Lemma 1.7 .

Due to the poor regularisation property of the operator $A$, the hypotheses on the nonlinearities are not easy to verify. A nontrivial example is given in the following corollary.

Corollary 2.7. Let $n=3,1<s<\frac{3}{2}, h \in C^{2}(\mathbb{R}, \mathbb{R}), f \in C\left(\mathbb{R}, H^{s-1}(\Omega)\right)$, and let $G$ be the Nemytskii operator induced by $h$. Then the assertions of Theorem 1.11 are true.

Proof. It merely remains to show that the substitution operator $G=(u \mapsto$ $h(u)) \in C^{1-}\left(H^{s+\frac{1}{2}}(\partial \Omega), H^{s-\frac{1}{2}}(\partial \Omega)\right)$ and is bounded on bounded sets. The boundary dimension is 2 and so we have the Sobolev embeddings $H^{s+\frac{1}{2}}(\partial \Omega) \hookrightarrow$ $B_{p p}^{1}(\partial \Omega) \hookrightarrow H^{s-\frac{1}{2}}(\partial \Omega)$ if $\frac{1}{2}<s<\frac{3}{2}$ and $s-\frac{1}{2}>1-2 / p>s-\frac{3}{2}$. We choose $2<p<$ $4 /(3-2 s)$ and observe that $G \in C^{1-}\left(B_{p p}^{1}(\partial \Omega), B_{p p}^{1}(\partial \Omega)\right)$, and is bounded on bounded sets [24, Lemma $4 \alpha$ of the appendix].

\section{Acknowledgment}

The author thanks the referee for pointing out the references [27-29]. 


\section{References}

1 S. Agmon. On the eigenfunctions and on the eigenvalues of general elliptic boundary value problems. Comm. Pure. Appl. Math. 15 (1962), 119-147.

2 S. Agmon, A. Douglis and L. Nirenberg. Estimates near the boundary for solutions of elliptic partial differential equations satisfying general boundary conditions II. Comm. Pure Appl. Math. 17 (1964), 35-92.

3 H. Amann. Gewöhnliche Differentialgleichungen (Berlin: Walter de Gruyter, 1983).

4 H. Amann. Existence and regularity for semilinear parabolic evolution equations. Ann. Scuola Norm. Sup. Pisa Cl. Sci. (4) 11 (1984), 593-676.

5 H. Amann. Semigroups and nonlinear evolution equations. Linear Algebra Appl. 84 (1986), 3-32.

6 H. Amann. Dynamic theory of quasilinear parabolic equations, part I: abstract evolution equations. J. Nonlinear Anal.: T., M. and A. 12 (1988), 895-919.

7 H. Amann. Dynamic theory of quasilinear parabolic equations, part II: reaction-diffusion-systems Diff. Int. Equations 3 (1990), 1-61.

8 J. Bergh and J. Löfström. Interpolation Spaces. An Introduction (Berlin: Springer, 1976).

9 R. Courant and D. Hilbert. Methoden der Mathematischen Physik II, 2 (Berlin: Springer, 1968).

10 J. I. Diaz and R. F. Jimenez. Aplication de la teoria no lineal de semigrupos a un operador pseudodifferential. Actas V II C.E.D.Y.A. (1984), 137-142.

11 A. Friedman and M. Shinbrot. The initial value problem for the linearized equations of water waves. J. Math. Mech. 17 (1968), 107-180.

12 R. M. Garipov. On the linear theory of gravity waves. Arch. Rational Mech. Anal. 24 (1967), 352-362.

13 K. Gröger. Initial boundary value problems from semiconductor device theory. Z. Angew. Math. Mech. 67 (1987), 345-355.

14 M. Grobbelaar-Van Dalsen. Semilinear evolution equations and fractional powers of a closed pair of operators. Proc. Roy. Soc. Edinburgh Sect. A 105 (1987), 101-115.

15 T. Hintermann. Evolutionsgleichungen mit dynamischen Randbedingungen (Dissertation, Universität Zürich, 1988).

16 L. Hörmander. The analysis of linear partial differential operators I (Berlin: Springer, 1985).

17 H. Lamb. Hydrodynamics, 4th edn (Cambridge: Cambridge University Press, 1916).

18 R. E. Langer. A problem in diffusion or in the flow of heat for a solid in contact with a fluid. Tôhoku Math. J. (2) 35 (1932), 260-275.

19 J. L. Lions. Quelques méthodes de résolutions des problèmes aux limites non linéaires (Paris: Dunod, 1969).

10 A. Pazy. Semigroups of linear operators and applications to partial differential equations (Berlin: Springer, 1983).

21 W. J. Rossouw. Behoudwetformulerings vir randvoorwaardes (Thesis, University of Pretoria, 1983).

22 N. Sauer. Linear evolution equations in two Banach spaces. Proc. Roy. Soc. Edinburgh Sect. A 91 (1982), 287-303.

23 R. Seeley. Interpolation in $L_{p}$ with boundary conditions. Studia. Math. 44 (1972), 47-60.

24 A. Stahel. Nichtlineare Randbedingungen bei Problemen vom Typ der Wellengleichung (Dissertation, Zürich, 1987).

$25 \mathrm{H}$. Triebel. Interpolation theory, function spaces, differential operators (Amsterdam: NorthHolland, 1978).

26 N. F. J. Van Rensburg. Dinamiese randvorwaardes vir parsiele differensiaalvergelykings (Thesis, University of Pretoria, 1982).

27 D. Lewis, J. Marsden and T. Ratiu. Formal stability of liquid drops. In Perspectives in Nonlinear Dynamics, eds Schlesinger, L., Cawley, J. P., Saenz, R. and Zachary, W. W. pp. 71-83 (World Scientific, 1986).

28 H. Okamoto. Nonstationary or stationary free boundary problems for perfect fluid with surface tension. In Recent Topics in Nonlinear PDE, Hiroshima 1983, pp. 143-154 (Amsterdam: North-Holland, 1983).

29 T. Benjamin and P. Olver. Hamiltonian structure, symmetries and conservation laws for water waves. J. Fluid Mech. 125 (1982), 137-185. 